\title{
Equivalent Activities of Repulsive Axon Guidance Receptors
}

\author{
Hong Long, Shingo Yoshikawa, and John B. Thomas \\ Molecular Neurobiology Laboratory, Salk Institute for Biological Studies, La Jolla, California 92037
}

Receptors on the growth cone at the leading edge of elongating axons play critical guidance roles by recognizing cues via their extracellular domains and transducing signals via their intracellular domains, resulting in changes in direction of growth. An important concept to have emerged in the axon guidance field is the importance of repulsion as a major guidance mechanism. Given the number and variety of different repulsive receptors, it is generally thought that there are likely to be qualitative differences in the signals they transduce. However, the nature of these possible differences is unknown. By creating chimeras using the extracellular and intracellular domains of three different Drosophila repulsive receptors, Unc5, Roundabout (Robo), and Derailed (Drl) and expressing them in defined cells within the embryonic nervous system, we examined the responses elicited by their intracellular domains systematically. Surprisingly, we found no qualitative differences in growth cone response or axon growth, suggesting that, despite their highly diverged sequences, each intracellular domain elicits repulsion via a common pathway. In terms of the signaling pathway(s) used by the repulsive receptors, mutations in the guanine nucleotide exchange factor Trio strongly enhance the repulsive activity of all three intracellular domains, suggesting that repulsion by Unc5, Robo, and Drl, and perhaps repulsion in general, involves Trio activity.

Key words: axon guidance; Drosophila; growth cone; receptor; repulsion; Trio

\section{Significance Statement}

A prevailing concept that has emerged in the axon guidance field is the importance of repulsion as a guidance mechanism for steering axons to their appropriate targets. Given the number and variety of different repulsive receptors, it is generally thought that there are differences in the signals that they transduce. However, this has never been tested directly. We have used the advanced genetics of Drosophila to compare directly the outputs of different repulsive receptors. Surprisingly, we found no qualitative differences in receptor-mediated repulsion, suggesting that, despite their highly diverged domain structure, each receptor couples to a common repulsive pathway. We went on to show that this common pathway involves Trio, a guanine nucleotide exchange factor known to promote cytoskeletal remodeling.

\section{Introduction}

Receptors on the growth cones of developing neurons recognize guidance cues in the environment and transduce signals that result in changes in direction of axon growth. A prevailing concept that has emerged in the axon guidance field is the importance of repulsion as a guidance cue in steering axons to their appropriate targets, as well as in generating maps of synaptic connectivity. There are several well characterized conserved repulsive guidance mechanisms, including Netrin signaling through the Unc5 family of receptors, Slit signaling through the family of Roundabout

\footnotetext{
Received Sept. 10, 2015; revised Nov. 12, 2015; accepted Dec. 7, 2015.

Author contributions: H.L. and J.B.T. designed research; H.L. and S.Y. performed research; S.Y. contributed unpublished reagents/analytic tools; H.L. and J.B.T. analyzed data; H.L. and J.B.T. wrote the paper.

This work was supported by National Institutes of Health (Grants NS040963 and DK077979 to J.B.T.) and a Canadian Institute of Health Research Fellowship to H.L. We thank Yi Leng for technical assistance.

The authors declare no competing financial interests.

Correspondence should be addressed to John B. Thomas, Molecular Neurobiology Laboratory, Salk Institute, 10010 North Torrey Pines Road, La Jolla, CA 92037. E-mail: jthomas@salk.edu.

DOI:10.1523/JNEUROSCI.3406-15.2016

Copyright $\odot 2016$ the authors $\quad 0270-6474 / 16 / 361140-11 \$ 15.00 / 0$
}

(Robo) receptors, Semaphorin signaling through Plexins/Neuropilins, and Wnt5 signaling through the Derailed (Drl)/Ryk receptors (for review, see O'Donnell et al., 2009; Bashaw and Klein, 2010; Kolodkin and Tessier-Lavigne, 2012). Despite the progress in uncovering these repulsive guidance mechanisms, the exact nature of repulsion remains elusive. The sheer number and variety of different repulsive receptors raises the possibility that there might be differences in the signals that they transduce; in fact, there is good evidence that, even within a family of receptors, one member cannot always be substituted for another (Spitzweck et al., 2010). However, the nature of any possible differences among repulsive receptors in terms of growth cone response is unknown.

Two key observations regarding the function of axon guidance receptors have been made over the past two decades. First, guidance receptors function in an appropriate manner (i.e., attraction vs repulsion) in many different types of neurons that do not normally express them, indicating that the intracellular signaling pathways to which they couple are likely present in most, if not all, neurons. Second, guidance receptors possess a modular structure in which the extracellular domain confers ligand spec- 

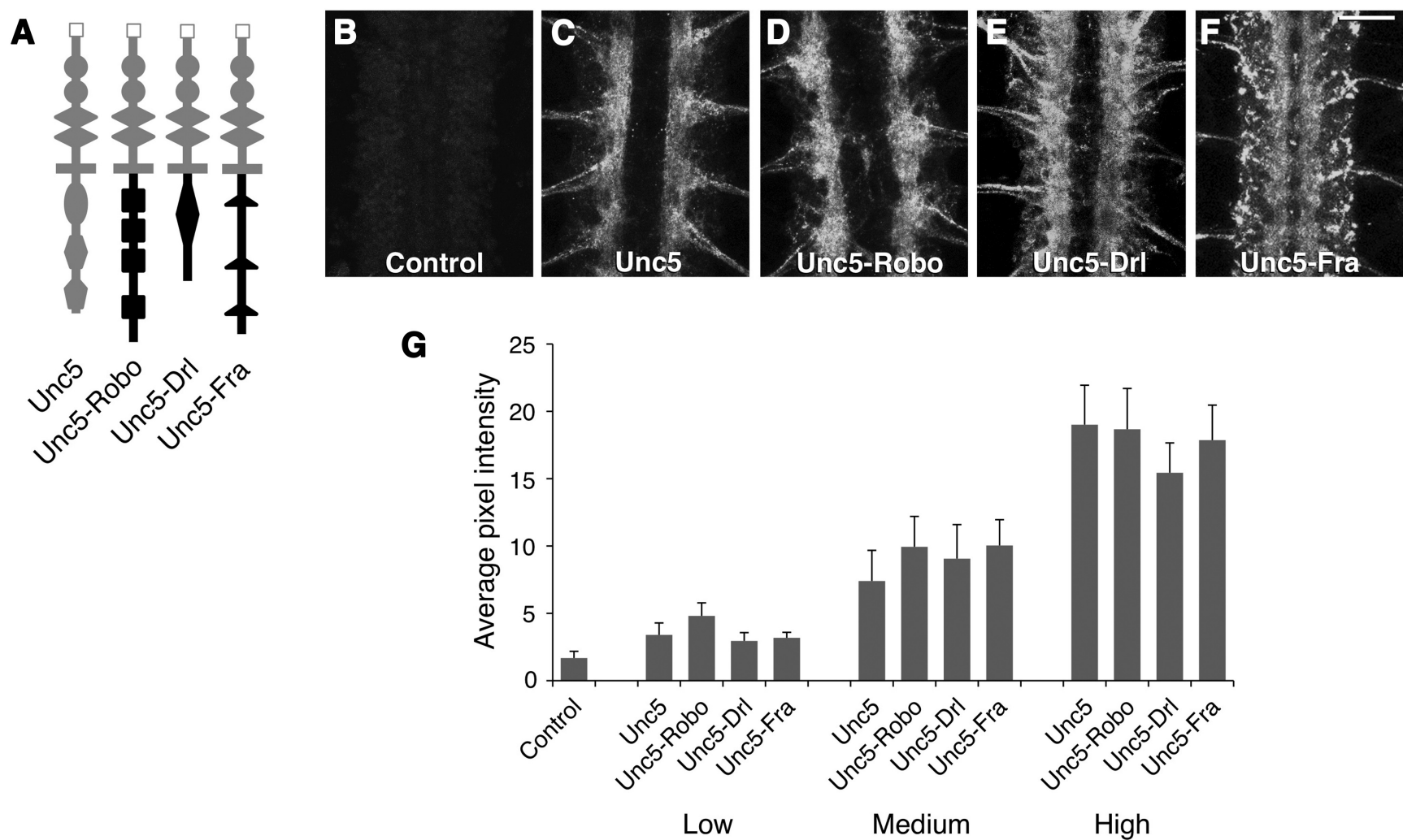

Figure 1. Chimeric receptors and their expression. $A$, Schematic showing Unc5, Unc5-Robo, Unc5-Drl, and Unc5-Fra protein domain structures. Each has an $\mathrm{HA}$ tag at the $\mathrm{N}$ terminus (open square). The Unc5 intracellular domain contains ZU5 (oval) and DB (hexagon) motifs plus a Death Domain (pentagon), Robo contains conserved CCO-CC3 domains (filled squares), Drl contains an atypical kinase domain (large diamond) and Fra contains conserved P1-P3 domains (triangles). $\boldsymbol{B}-\boldsymbol{F}$, Anti-HA antibody staining of live, dissected, unfixed E16 embryonic VNCs. $\boldsymbol{B}$, No specific staining revealed in the control. $\mathbf{C}-\boldsymbol{F}$, Pan-neuronal membrane expression detected in medium-level expressors of Unc5 (C), Unc5-Robo (D), Unc5-Drl (E), and Unc5-Fra $(\boldsymbol{F})$. $\boldsymbol{G}$, For each receptor, low-, medium-, and high-level expressors are selected based on their protein expression levels. Number of embryos examined in G: Control ( $n=15)$, Unc5 Low ( $n=6)$, Unc5-Robo Low ( $n=7)$, Unc5-Drl Low $(n=9)$, Unc5-Fra Low $(n=9)$, Unc5 Medium ( $n=39)$, Unc5-Robo Medium $(n=22)$, Unc5-Drl Medium $(n=66)$, Unc5-Fra Medium $(n=16)$, Unc5 High $(n=8)$, Unc5-Robo High $(n=8)$, Unc5-Drl High $(n=6)$, Unc5-Fra High $(n=11)$. Error bars indicate SD. Genotypes: $\boldsymbol{B},+/+;$ scrt-GAL4/+; C, UAS-Unc5 Medium/+; scrt-GAL4/+; $\boldsymbol{D}$, UAS-Unc5-robo Medium/+; scrt-GAL4/+; $\boldsymbol{E}$, UAS-Unc5-drI Medium/+; scrt-GAL4/+;F, UAS-Unc5-fra Medium/+;scrt-GAL4/+. Scale bar, $20 \mu \mathrm{m}$.

ificity whereas the intracellular domain dictates the response (Bashaw and Goodman, 1999; Keleman and Dickson, 2001; Stein et al., 2001; Evans and Bashaw, 2010; Spitzweck et al., 2010).

The set of repulsive axon guidance receptors is composed of a number of different families with unrelated cytoplasmic domains. For example, Unc5 contains ZU5 and DB motifs plus a Death Domain, Robo contains conserved CC0-CC3 domains, and Drl contains an atypical kinase domain plus a PDZ binding motif. Given their different cytoplasmic domains, do these receptors engage different intracellular signaling pathways? Are there actually differences between Robo-, Unc5-, and Drl-mediated repulsion? To date, no systematic study addressing these questions has been performed.

To compare directly the responses elicited by Unc5, Robo, and Drl and to begin identifying the signaling components downstream of each, we created chimeras consisting of the Netrinbinding Unc5 extracellular domain fused to the intracellular domains of Robo and Drl. By expressing Unc5, Unc5-Robo, and Unc5-Drl in specific neurons, we were able to assay in detail the responses elicited by each of the intracellular domains to the Netrin source at the midline of the nervous system. Surprisingly, we found no qualitative differences in growth cone response or axon growth elicited by the three intracellular domains. In addition, when fused to the Drl extracellular domain, the Unc5 and Robo intracellular domains were able to repel axons in a manner indistinguishable from that of Drl and, when fused to the Robo extracellular domain, the Unc5 and Drl intracellular domains were able to repel axons similarly to Robo itself. In terms of the downstream signal transduction pathways used by the receptors, mutations in the guanine nucleotide exchange factor Trio strongly enhance the repulsive activity of all three intracellular domains, suggesting that repulsion elicited by Unc5, Robo, and Drl involves Trio activity.

\section{Materials and Methods}

Fly strains and genetics. The fly lines used were scrt-GAL4 (Boyle et al., 2006), dMP2-GAL4 on the second chromosome (Miguel-Aliaga and Thor, 2004), eagle-GAL4 (Dittrich et al., 1997), ap-GAL4 (Calleja et al., 1996), UAS-GAP-myc-GFP (Callahan et al., 1998), UAS-tau-myc-GFP, UAS-drl (Bonkowsky et al., 1999), and NetrinAB ${ }^{\Delta}$ (Brankatschk and Dickson, 2006). UAS-Unc5 lines and UAS-robo lines with different expression levels were generated by mobilizing the original lines (Kidd et al., 1998; Keleman and Dickson, 2001) with $\Delta 2-3$ transposase. robo ${ }^{1}$, C155-GAL4, trio $^{\mathrm{S} 137203}$ and trio ${ }^{6 \mathrm{~A}}$ were from the Bloomington Stock Center. Transheterozygous trio ${ }^{\mathrm{S} 137203} /$ trio $^{6 \mathrm{~A}}$ individuals were used for trio zygotic mutant analysis. Flies of either sex were used for all experiments except Netrin $A B^{\Delta}$ mutants, which were hemizygous males.

Fly germline transformation was performed as described previously (Rubin and Spradling, 1982). For each chimera, multiple lines were generated. The expression level of each line containing the Unc5 extracellular domain was examined by antibody staining. High-level expression was achieved by combinations of multiple lines. Flies were raised at $25^{\circ} \mathrm{C}$. Embryos were kept at $29^{\circ} \mathrm{C}$ for appropriate expression of transgenes. 
Constructs. All constructs were generated using standard PCR-based cloning procedures. UAS-HA-Unc5 (Keleman and Dickson, 2001) was used to generate UAS-HA-Unc5-robo, UAS-HA-Unc5-drl, and UAS-HA-Unc5-fra. The HindIII- XbaI DNA fragment of UAS-HAUnc5, which corresponds to the Unc5 cytoplasmic domain, was replaced by the cytoplasmic domain DNA fragments of robo, $d r l$, and fra, respectively. As a result, UAS-HA-Unc5-robo encodes the protein: Wingless aa1-32, Ala-Arg, $3 \times$ HA, Ser-Leu-Asp, Unc5 aa32-536, Ser-Phe, Robo aa940-1395; UAS-HA-Unc5-drl encodes: Wingless aa1-32, Ala-Arg, $3 \times \mathrm{HA}$, SerLeu-Asp, Unc5 aa32-536, Ser-Phe, Drl aa265610; and UAS-HA-Unc5-fra encodes: Wingless aa1-32, Ala-Arg, 3×HA, Ser-Leu-Asp, Unc5 aa32-536, Ser-Phe, Fra aa1101-1374.

To build UAS-HA-robo-Unc5 and UAS-HArobo-drl, the HA-robo extracellular and transmembrane domain DNA flanked by EcoRI and HindIII sites was derived from the robo knock-in construct (Spitzweck et al., 2010). This fragment was ligated together with the cytoplasmic domain DNA fragment of either Unc5 or $d r l$ into the pUAST vector (Brand and Perrimon, 1993). As a result, UAS-HA-roboUnc5 encodes a protein: Wingless aa 1-32, AlaArg, 7×HA, Robo aa52-939, Leu-Ser-PheArg, Unc5 aa537-1072; UAS-HA-robo-drl encodes a protein: Wingless aa1-32, Ala-Arg, $7 \times$ HA, Robo aa52-939, Leu-Ser-Phe, Drl aa265-610.

The extracellular and transmembrane domain DNA fragment flanked by EcoRI and HindIII sites of $d r l$ was derived from the $d r l$ 3.1cDNA construct (Callahan et al., 1995). This fragment was ligated with either robo or Unc5 cytoplasmic domain DNA fragment flanked by HindIII and XbaI sites into the pUAST vector. As a result, UAS-drl-robo encodes a protein: Drl aa1-264, Lys-Leu, Robo aa940-1395; UAS- drl-Unc5 encodes a protein: Drl aal-264, Ser-Phe-Arg, Unc5 aa537-1072. The junction sites of all constructs have been sequenced.

Immunohistochemistry. All embryos were processed for immunohistochemistry. Embryos were dissected and stained as described previ-

ously (Callahan et al., 1995). Primary antibodies used: monoclonal (mAb) anti-GFP 3E10; rabbit polyclonal anti-GFP (Life Technologies); $\mathrm{mAb}$ BP102; mAb anti-Fas2 (Developmental Studies Hybridoma Bank); Cy5conjugated anti-HRP (Jackson ImmunoResearch); and rat mAb anti-HA 3F10 (Roche).

For live antibody staining, dissected, unfixed embryos were incubated with anti-HA 3F10 diluted in 5\% NGS in PBS for $2 \mathrm{~h}$. After incubation, embryos were washed and fixed in paraformaldehyde followed by secondary antibody detection. No detergent was used throughout the staining process.

Receptor protein expression and quantification of $d M P 2$ phenotypes. After anti-HA staining, samples were imaged by confocal microscopy. A rectangle encompassing A1-A3 within an individual ventral nerve cord (VNC) was drawn. The mean pixel intensity within the rectangle was measured with Zeiss ZEN software. For each genotype, multiple embryos were examined. For quantification of dMP2 phenotypes, dMP2 neurons in segments T1-A7 of stage 16 embryos were assayed. To measure the dMP2 axon length at stage E15-E16, Z-stack images were taken from the cell body to the growth cone; the images were flattened and the axon length from the hillock to the tip of the growth cone was measured.
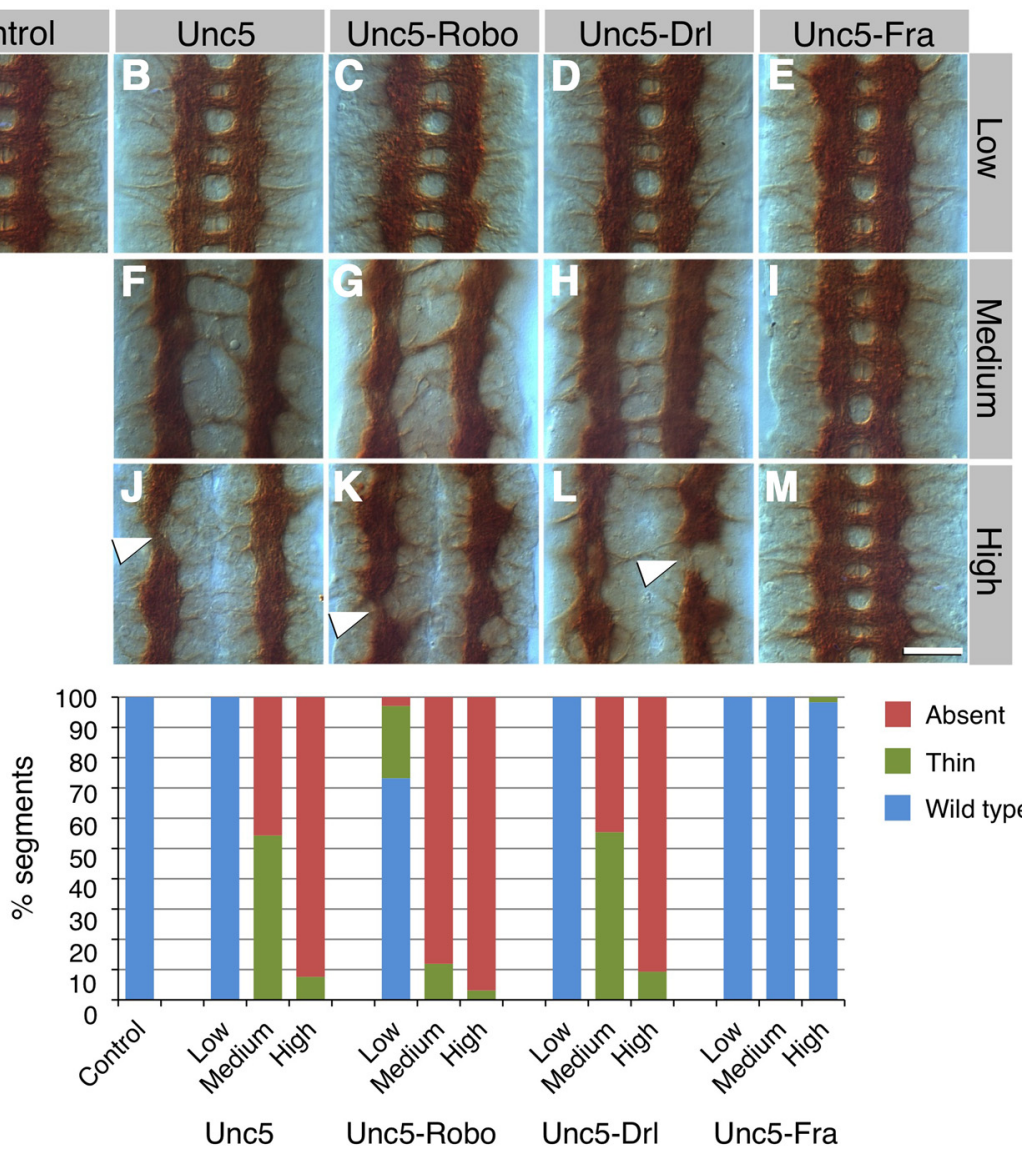

Absent

Thin

Wild type

Figure 2. Pan-neuronal expression of chimeras prevents axons from crossing the midline. $\boldsymbol{A}-\boldsymbol{M}$, Late-stage embryonic VNC stained with BP102 mAb. $\boldsymbol{B}-\boldsymbol{E}$, Embryos expressing low levels of receptors have largely intact axon projection patterns compared

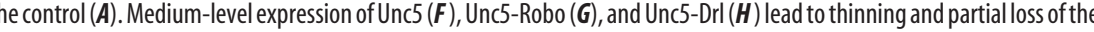
commissures, whereas high-level expression $(\boldsymbol{J}-\boldsymbol{L}$ ) eliminates commissure formation. Note the relocation of connectives further from the midline in $\boldsymbol{F}-\boldsymbol{H}, \boldsymbol{J}-\boldsymbol{L}$, and the thinning and broken connectives in $\boldsymbol{J}-\boldsymbol{L}$ (arrowheads). In contrast, Unc5-Fra does not prevent ed, percentage of embryos showing phenotype, number of segments examined: Control ( $n=44,0 \%, n=352)$, Unc5 Low ( $n=$ , 144), Unc5 Medium ( $n=31,100 \%, n=247)$, Unc5 High $(n=23,100 \%, n=184)$, Unc5-Robo Low $(n=20,100 \%$, $n=138)$, Unc5-Robo Medium $(n=21,100 \%, n=168)$, Unc5-Robo High $(n=34,100 \%, n=295)$, Unc5-Drl Low $(n=14,0 \%$, One copy of scrt-GAL4 plus one copy of corresponding receptor transgene with either low or medium expression levels; J-M, 0 ne copy of scrt-GAL4 plus two copies of corresponding receptor transgenes. Scale bar, $20 \mu \mathrm{m}$.

\section{Results}

\section{Unc5 extracellular domain chimeras}

The earliest differentiating neurons within the Drosophila embryo form an initial framework of axons that comprise the major tracts within the CNS: the bilaterally symmetric longitudinal connectives, which run the length of the CNS, and an anterior and posterior commissure (AC and PC) connecting the two sides of each segment. A set of midline glia divide the two halves of the nervous system and play a critical role in axon trafficking. The midline glia secrete $\mathrm{Ne}$ trins, diffusible factors that are capable of attracting contralaterally projecting axons that express the receptor Frazzled (Fra) or repelling axons that express the receptor Unc5 (Hedgecock et al., 1990; Hong et al., 1999; Keleman and Dickson, 2001; Labrador et al., 2005). The extracellular domains of both Fra and Unc5 bind Netrin; the response, whether attractive or repulsive, is dictated by the Fra or Unc5 intracellular domain, respectively (Keleman and Dickson, 2001).

Chimeras consisting of the Unc5 extracellular and transmembrane domains fused to the intracellular domains of Robo and 


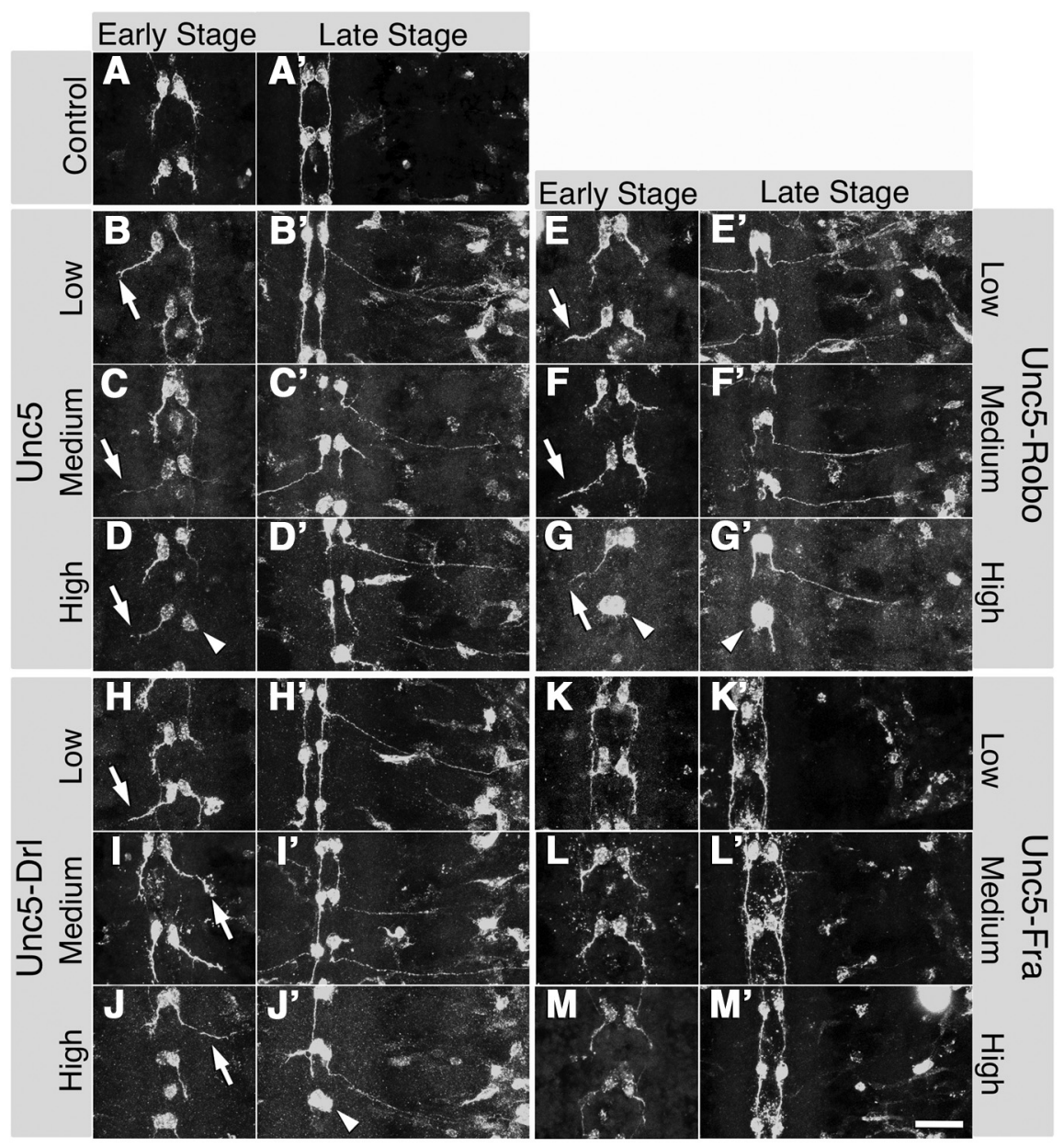

Figure 3. Effects of chimeras on the dMP2 growth cone and axon. dMP2 neurons are labeled by dMP2-GAL4 driving membranetargeted GFP. Early (E14) and late (E16) stage embryos of each genotype are shown. $\boldsymbol{A}$ and $\boldsymbol{A}^{\prime}$, Control. $\boldsymbol{B}-\boldsymbol{D}^{\prime}$, Unc5, low to high expression. $\boldsymbol{E}-\boldsymbol{G}^{\prime}$, Unc5-Robo, low to high expression. $\boldsymbol{H}-\boldsymbol{J}^{\prime}$, Unc5-Drl, low to high expression. At early-stage E14, the axons of dMP2 neurons expressing Unc5, Unc5-Robo, and Unc5-Drl are oriented away from the midline (arrows). With increasing expression levels, some neurons fail to extend axons (arrowheads). $\boldsymbol{K}-\boldsymbol{M}^{\prime}$, Unc5-Fra, low to high expression. In contrast to Unc5, Unc5-Robo, and Unc5-Drl, Unc5-Fra expression has no effect on dMP2 axons. Genotypes: $\boldsymbol{A}$, dMP2-GAL4/+; UAS-GAP-myc$G F P /+; \boldsymbol{B}-\boldsymbol{C}^{\prime}, \boldsymbol{E}-\boldsymbol{F}^{\prime}, \boldsymbol{H}-\boldsymbol{I}^{\prime}, \boldsymbol{K}-\boldsymbol{L}^{\prime}$, One copy of $d M P 2-G A L 4$, one copy of UAS-GAP-myc-GFP, plus one copy of corresponding receptor transgene with low or medium expression levels; $\mathbf{D}, \mathbf{D}^{\prime}, \mathbf{G}, \mathbf{G}^{\prime}, \boldsymbol{J}, \mathbf{J}^{\prime}, \mathbf{M}^{\prime}, \mathbf{M}^{\prime}$, One copy of dMP2-GAL4, one copy of UAS-GAP-myc-GFP, plus two copies of corresponding receptor transgenes. Scale bar, $20 \mu \mathrm{m}$.

Drl were created, producing Unc5-Robo and Unc5-Drl, respectively (Fig. 1A). For each chimera, multiple UAS transformants were generated using pUAST (Brand and Perrimon, 1993). We chose this method rather than inserting each into a defined genomic location by site-specific recombination (Groth et al., 2004) because we reasoned it would be useful to have several independent transgenes on different chromosomes comprising a range of expression levels. Ten independent transformants were isolated for each chimera. For Unc5 itself, previously created UAS-Unc5 insertion was mobilized (Keleman and Dickson, 2001) to generate insertions with a range of expression levels. To monitor expression, the Unc5 extracellular domain chimeras, as well as Unc5 itself, were HA epitope tagged immediately downstream of the signal sequence, a position known not to affect function (Keleman and Dickson, 2001).

In addition to the Unc5 extracellular domain chimeras using Robo and Drl intracellular domains, Unc5-Fra was constructed as a control. The Fra intracellular domain has been shown previously to confer attraction when substituted for the intracellular domain of either Unc5 or Robo (Bashaw and Goodman, 1999;
Keleman and Dickson, 2001). Unc5-Fra was also HA epitope tagged identically to Unc5-Robo and Unc5-Drl.

Unc5, Unc5-Robo, Unc5-Drl, and Unc5Fra were expressed in the CNS using the GAL4/UAS system (Brand and Perrimon, 1993), which capitalizes on the ability of the yeast GAL4 transcriptional activator to transcribe a gene of interest placed downstream of UAS binding sites. We first examined the expression of Unc5 and the chimeras by live antibody staining against the $\mathrm{N}$-terminal HA tag to be certain that the receptors were properly inserted into the cell membrane and to quantify the expression levels of receptors at the cell surface. When driven by the scrt-GAL4 panneuronal driver, Unc5 and all of the chimeras were predominantly localized on the surface of axons (Fig. $1 B-F$ ), although Unc5-Fra showed higher levels on the surface of cell bodies compared with Unc5, Unc5-Robo, and Unc5-Drl. We quantified the expression level of each transgenic line and created combinations of individual lines to achieve a variety of expression levels. Based on expression levels, specific lines or combinations of lines were carefully matched and divided into three different expression groups for each chimera: low, medium, and high. (Fig. 1G).

\section{Repulsion elicited by pan-neuronal expression of Unc5, Unc5-Robo, and Unc5-Drl}

We initially tested Unc5, Unc5-Robo, and Unc5-Drl for activity by expressing them pan-neuronally using scrt-GAL4 and visualizing the overall structure of the CNS with the pan-axonal BP102 antibody (Seeger et al., 1993). Both chimeras showed general repulsive activity similar to the native Unc5 receptor (Fig. 2).

Low-level pan-neuronal expression of Unc5, Unc5-Robo, or Unc5-Drl results in little or no phenotype, with Unc5-Robo showing slight thinning of the commissures in $23 \%$ of segments (Fig. $2 B-D, N$ ). Medium-level pan-neuronal expression of Unc5-Robo or Unc5-Drl caused markedly reduced midline crossing, resulting in reduction of commissures in virtually every segment, plus axons projecting out of the CNS, similar to matched medium levels of pan-neuronally expressed Unc5 itself (Fig. $2 F-H, N$; see also Keleman and Dickson, 2001). High-level expression of Unc5, Unc5-Robo, or Unc5-Drl results in an essentially commissureless phenotype consisting of missing commissures in $>90 \%$ of segments (Fig. $2 J-L, N)$. The longitudinal connectives of individuals with medium and high levels of expression are located further from the midline within the VNC and, in many segments of the high expressors, the connectives are thinner or broken, suggesting the presence of stalled axons (Fig. 2, arrowheads). Collectively, these results indicate that the two chimeras have repulsive 
A

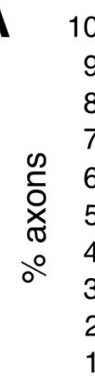

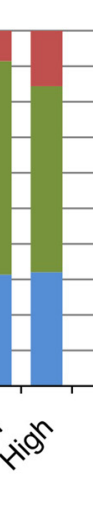

Unc5

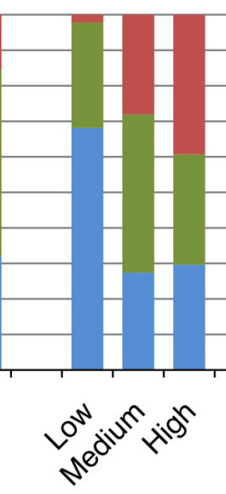

Unc5-Robo
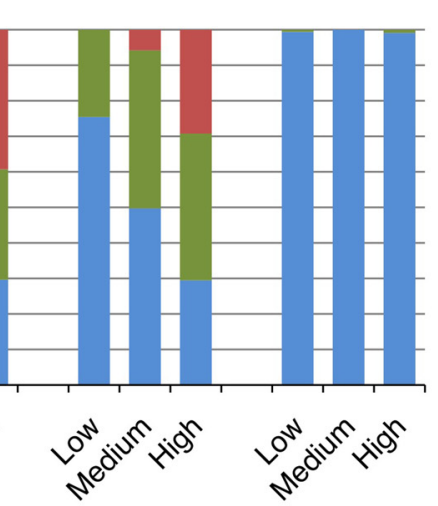

Unc5-Drl

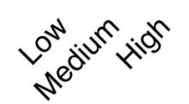

Unc5-Fra

B

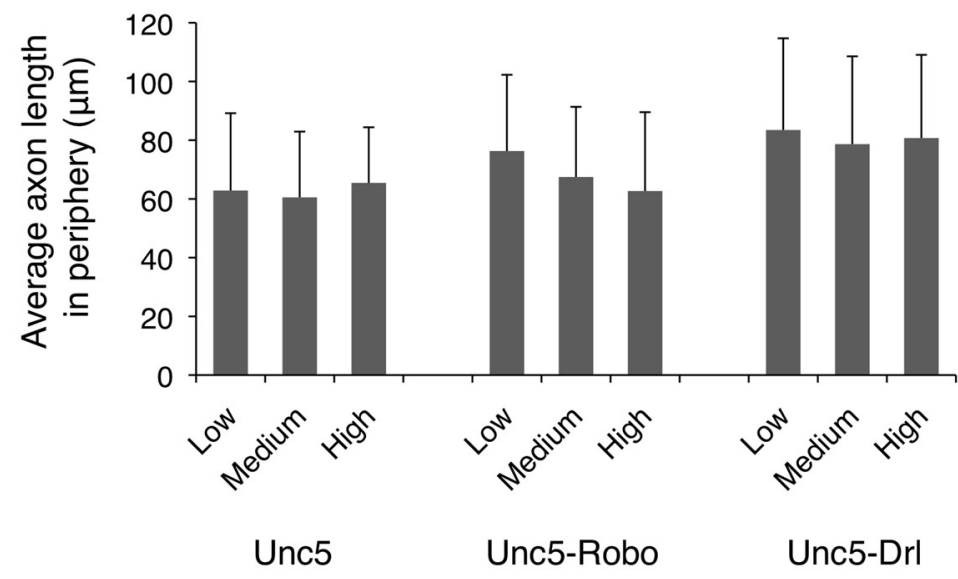

Figure 4. Quantification of dMP2 axon behavior shown in Figure 3.A, Neuron behaviors are grouped into three categories: axons that project posteriorly as in wild-type, axons that exit the VNC, and axons that are severely stalled or unextended altogether. Expression of Unc5, Unc5-Robo, or Unc5-Drl forces axons to exit the VNC. Increased expression of these receptors increases axon exiting and stalling. $\boldsymbol{B}$, Average length of dMP2 axons projecting into the periphery at stage E16 for Unc5, Unc5-Robo, and Unc5-Drl at different expression levels. $\boldsymbol{A}$, Number of embryos examined, percentage of embryos showing phenotype, number of hemisegments examined: Control $(n=36,0 \%, n=200)$, Unc5 Low $(n=10,60 \%, n=96)$, Unc5 Medium ( $n=49,100 \%, n=416)$, Unc5 High $(n=20,100 \%, n=224)$, Unc5-Robo Low $(n=14,100 \%, n=138)$, Unc5-Robo Medium ( $n=54,100 \%, n=512)$, Unc5-Robo High ( $n=30,100 \%, n=368)$, Unc5-Drl Low ( $n=47,100 \%$, $n=624)$, Unc5-Drl Medium ( $n=102,100 \%, n=1072)$, Unc5-Drl High $(n=50,100 \%, n=720)$, Unc5-Fra Low $(n=40,3 \%, n=160)$, Unc5-Fra Medium $(n=16,0 \%, n=144)$, Unc5-Fra High $(n=9,11 \%, n=112)$. $\boldsymbol{B}$, Number of axons examined: Unc5 Low $(n=7)$, Unc5 Medium $(n=96)$, Unc5 High $(n=91)$, Unc5-Robo Low $(n=71)$, Unc5-Robo Medium ( $n=76)$, Unc5-Robo High $(n=65)$, Unc5-Drl Low $(n=113)$, Unc5-Drl Medium $(n=151)$, Unc5-Drl High $(n=172)$. Error bars indicate SD. Genotypes are described in Figure 3 legend.

activities similar to Unc5 in repelling axons away from the midline.

In contrast to Unc5-Robo and Unc5-Drl, Unc5-Fra produced an overall axon projection pattern resembling wild-type when pan-neuronally expressed (Fig. 2E, I,M); however, like Fra itself (Yang et al., 2009), Unc5-Fra is capable of directing some axons to the midline when expressed by certain ipsilaterally projecting neurons. This lack of strong effect of Unc5-Fra expression is consistent with previous studies (Keleman and Dickson, 2001) and is likely due to the fact that endogenous Fra is normally expressed by the majority of neurons within the CNS (Kolodziej et al., 1996). This result also reinforces the idea that the repulsive responses generated by Unc5-Robo and Unc5-Drl are mediated through their cytoplasmic domains, not the Unc5 extracellular domain.

\section{Repulsion of the dMP2 axon elicited by Unc5, Unc5-Robo,} and Unc5-Drl

To examine the effects of the chimeras on the orientation and outgrowth of individual axons, we expressed them in the dMP2 neuron using the $A M P 2-G A L 4$ driver and visualized the dMP2 growth cones and axons with UAS-GAP-myc-GFP (Callahan et al., 1998; Fig. 3). dMP2 is an early developing neuron that projects its axon posteriorly in the ipsilateral connective, fasciculating with its homolog in the adjacent segment (Thomas et al., 1984; Miguel-Aliaga and Thor, 2004). dMP2 normally expresses Fra and Robo, but not Drl or Unc5. In addition, dMP2 does not express Commissureless (Comm), which functions in commissural neurons to downregulate Robo (Keleman et al., 2002; Keleman et al., 2005).

When forced to express Unc5, Unc5-Robo, or Unc5-Drl at matched medium levels, the dMP2 growth cones orient away from the midline in $\sim 60 \%, 45 \%$, and $45 \%$, of hemisegments, respectively (Figs. 3,4A), in contrast to $0 \%$ for controls or for dMP2s expressing Unc5-Fra. Therefore, like Unc5, both Unc5Robo and Unc5-Drl are capable of reorienting growth cones away from the midline, consistent with the pan-neuronal expression phenotype (Fig. 2).

When expressed at medium levels in $\mathrm{dMP} 2, \mathrm{Unc5}$ and the two repulsive chimeras show quantitative differences in their effects. For example, Unc5-Robo shows a relatively high percentage of dMP2s with no axons compared with Unc5 and Unc5-Drl (Figs. $3,4 A$ ). These differences could reflect the possibility that the different intracellular domains engage different signaling pathways. 


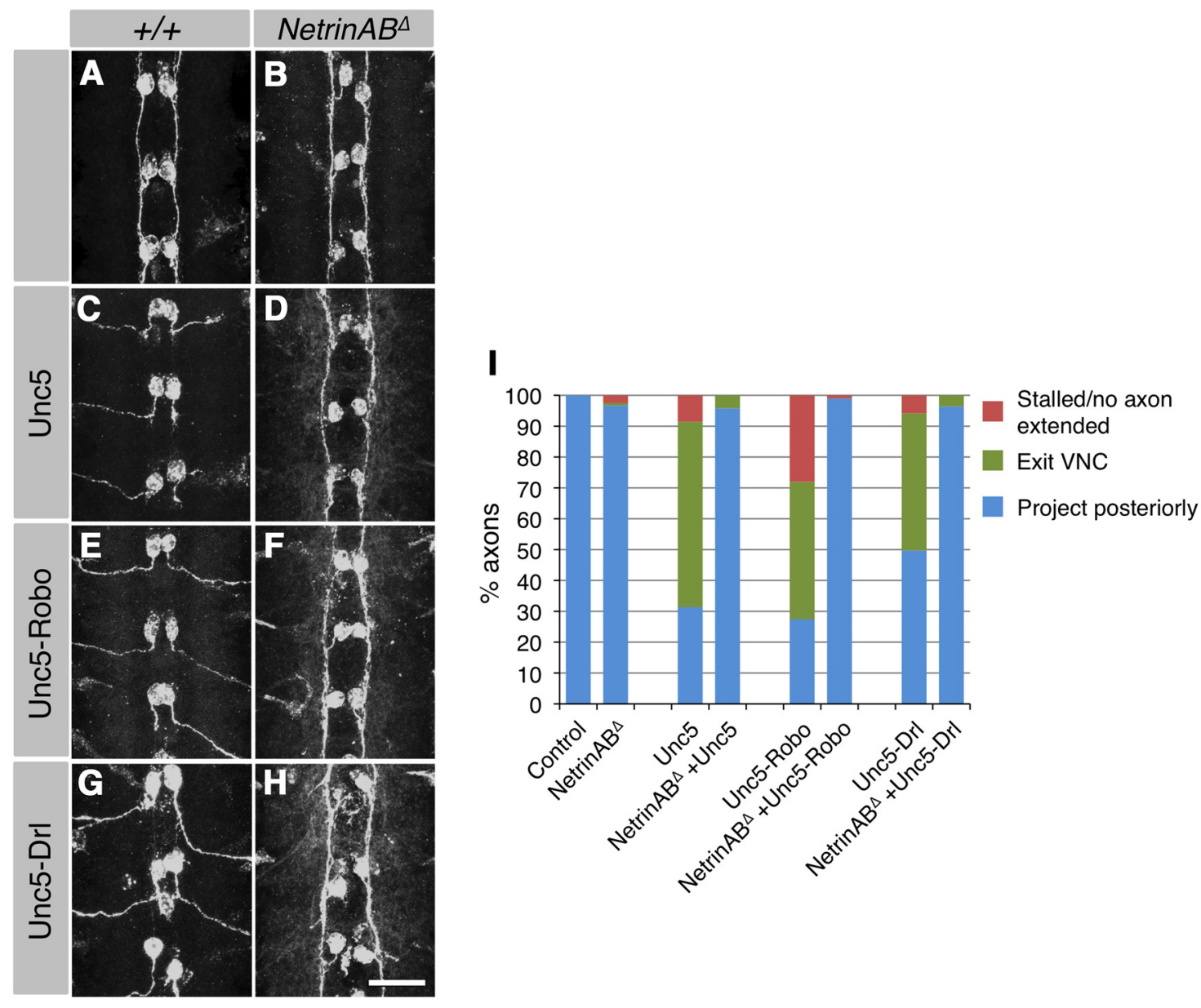

Figure 5. Repulsive activities of Unc5, Unc5-Robo, and Unc5-Drl are Netrin dependent. Axon exiting and stalling phenotypes are completely suppressed by NetrinAB ${ }^{\Delta}$. Normal dMP2 axon projection patterns in control $(\boldsymbol{A})$ and NetrinA $B^{\Delta}$ mutant $(\boldsymbol{B})$. dMP2 neurons expressing medium levels of Unc5, Unc5-Robo, and Unc5-Drl in the control background $(\boldsymbol{C}, \boldsymbol{E}, \boldsymbol{G})$ and in a NetrinAB ${ }^{\Delta}$ mutant background $(\boldsymbol{D}, \boldsymbol{F}, \boldsymbol{H})$. Quantification of dMP2 axon phenotypes shown in $I$. Neuron behaviors are grouped as in Figure $4 A$. Number of embryos examined, percentage of embryos showing phenotype, number of hemisegments examined: NetrinAB ${ }^{\Delta}(n=20,10 \%, n=280)$, NetrinAB ${ }^{\Delta}+U n c 5(n=6,16 \%, n=108)$, NetrinAB ${ }^{\Delta}+$ Unc5-Robo $(n=17,6 \%, n=102), N e t r i n A B{ }^{\Delta}$ $+\operatorname{Unc5}$-Drl $(n=14,7 \%, n=84)$. Numbers for Control, Unc5, Unc5-Robo, and Unc5-Drl are indicated in the Figure 4 legend. Genotypes: $A, d M P 2-G A L 4 /+; U A S-G A P-m y c-G F P /+; B, N e t r i n A B{ }^{\Delta} / Y$; dMP2-GAL4/+; UAS-GAP-myc-GFP/+;C, dMP2-GAL4/UAS-UnC5 Medium; UAS-GAP-myc-GFP/+; D, NetrinAB ${ }^{\Delta} /$; ; dMP2-GAL4/UAS-Unc5 Medium; UAS-GAP-myc-GFP/+; E, dMP2-GAL4/UASUnc5-robo Medium; UAS-GAP-myc-GFP/+; $\boldsymbol{F}$, NetrinAB ${ }^{\Delta} / Y$; dMP2-GAL4/UAS-Unc5-robo Medium; UAS-GAP-myc-GFP/+; G, dMP2-GAL4/UAS-Unc5-drl Medium; UAS-GAP-myc-GFP/+; $\boldsymbol{H}$, NetrinAB ${ }^{\Delta} / Y$; dMP2-GAL4/UAS-Unc5-drl Medium; UAS-GAP-myc-GFP/+. Scale bar, $20 \mu \mathrm{m}$.

Alternatively, all three intracellular domains may engage the same pathway, but differ in their efficiency in coupling to it. To address this, in addition to medium levels, we expressed matched low and high levels of Unc5, Unc5-Robo, and Unc5Drl in the dMP2 neurons. We found that, for Unc5 and the two repulsive chimeras, increasing their levels of expression resulted in increased numbers of axons exiting the CNS, followed by increased numbers of shortened and finally completely stalled axons within the CNS (Fig. $4 A$ ), whereas Unc5-Fra had little or no effect at any expression level. Therefore, when a range of expression levels are examined, any differences in axon guidance responses elicited by the three intracellular domains appear to be quantitative.

For those dMP2 neurons that extend axons out of the VNC and into the periphery, where they presumably encounter less of the Netrin signal, there is no evidence of stalling and the average axon lengths are similar between the different repulsive receptors regardless of expression level (Fig. $4 B$ ). This suggests that the responses of dMP2 elicited by expression of the repulsive receptors are dependent on Netrin.

\section{Repulsive responses are Netrin dependent}

The phenotypes observed for misexpression of Unc5, Unc5-Robo, and Unc5-Drl are consistent with the notion that these receptors transduce repulsive signals in response to midline-secreted Netrins. To confirm the involvement of the Netrins, we expressed medium levels of Unc5, Unc5-Robo, and Unc5-Drl specifically in dMP2 neurons in a Netrin mutant $\left(\mathrm{Net} A B^{\Delta}\right)$ background (Fig. 5). In the absence of Netrins, Unc5, Unc5-Robo, or Unc5-Drl were no longer able to repel dMP2 axons away from the midline (Fig. 5D, F, H,I), similar to what has been shown previously for Unc5-mediated repulsion (Keleman and Dickson, 2001). In addition, the severe axonstalling phenotype resulting from high levels of receptor expression is also completely suppressed. This complete suppression indicates that both Unc5-Robo and Unc5-Drl, like Unc5 itself, function as receptors to transduce the Netrin signal into a repulsive response and that Netrins appear to be the sole ligands mediating this response.

\section{Repulsive responses generated in different types of neurons}

To address the universality of the repulsive responses generated by Unc5, Unc5-Robo, and Unc5-Drl, we examined the responses 

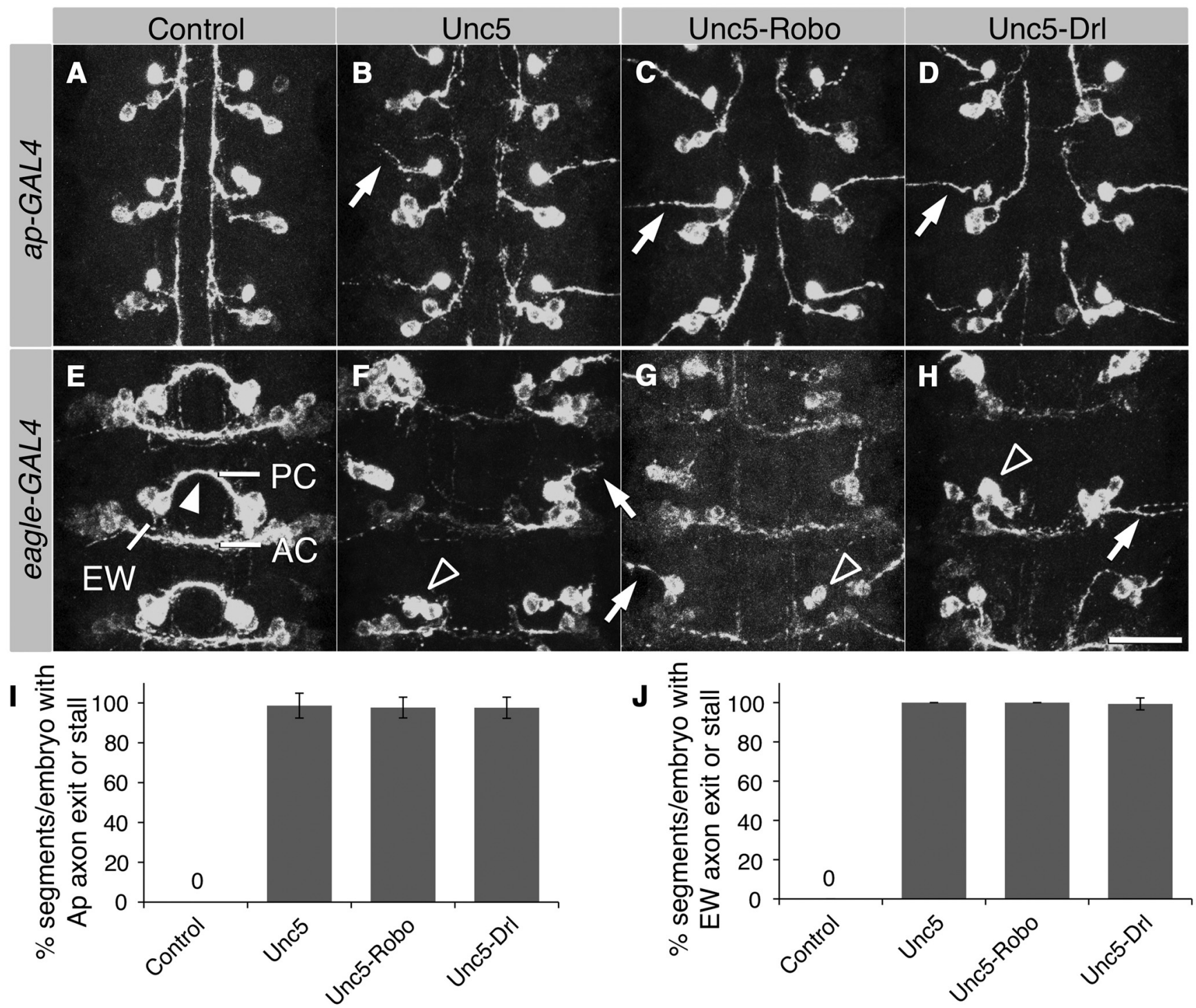

Figure 6. Repulsive responses generated by Unc5 and chimera expression in the Ap and Eagle neurons. $\boldsymbol{A}-\boldsymbol{D}$, Compared with control (A), expression of medium-level repulsive receptors in Ap neurons results in axons exiting the VNC (arrows). Quantification shown in I.E-H, Repulsive responses generated in EW neurons. eagle-GAL4 drives early and reproducibly high expression in the EW neurons, which cross the midline in the $\mathrm{PC}$ (filled arrowhead in $\boldsymbol{E}$ ), and late, stochastic expression in neurons, which cross the midline in the AC. The expression of medium repulsive receptors prevents EW axons from crossing the midline. Exiting axons (arrows) and neurons with no axon (open arrowheads) are also observed. Quantification shown in $J$. Number of embryos examined, percentage of embryos showing phenotype, number of segments examined: I, Control $(n=14,0 \%, n=98)$, Unc5 ( $n=21,100 \%, n=151)$, Unc5-Robo $(n=23,100 \%, n=162)$, Unc5-Drl ( $n=28,100 \%$, $n=205)$; J, Control $(n=19,0 \%, n=144)$, Unc5 ( $n=23,100 \%, n=157)$, Unc5-Robo $(n=27,100 \%, n=186)$, Unc5-Drl ( $n=22,100 \%, n=147)$. Genotypes:A, ap-GAL4 UAS-tau-myc-GFP/+; B, ap-GAL4 UAS-tau-myc-GFP/UAS-Unc5 Medium; C, ap-GAL4 UAS-tau-myc-GFP/UAS-Unc5-robo Medium; $\boldsymbol{D}$, ap-GAL4 UAS-tau-myc-GFP/UAS-Unc5-drI Medium; $\boldsymbol{E}$, UAS-tau-myc-GFP/+; eagleGAL4/+;F, UAS-tau-myc-GFP/UAS-Unc5 Medium;eagle-GAL4/+;G,UAS-tau-myc-GFP/UAS-Unc5-robo Medium;eagle-GAL4/+; H, UAS-tau-myc-GFP/UAS-Unc5-drIMedium; eagle-GAL4/+. Scale bar, $20 \mu \mathrm{m}$

elicited by their expression in the dorsal Ap neurons (Lundgren et al., 1995; O'Keefe et al., 1998) and the Eagle-expressing EW neurons (Dittrich et al., 1997; Yoshikawa et al., 2003). The dorsal Ap neurons extend axons ipsilaterally and anteriorly, whereas the EW neurons extend axons across the midline in the PC. Similar to the response of the $\mathrm{dMP} 2$ axons, the Ap and EW axons orient away from the midline when forced to express Unc5, Unc5-Robo, or Unc5-Drl (Fig. 6). Therefore, each intracellular domain is capable of eliciting repulsion in a variety of different types of neurons.

Repulsive activity of the intracellular domains when fused to the Drl and Robo extracellular domains

To assess whether the repulsive intracellular domains would repel axons when fused to a different extracellular domain binding a different ligand, we fused the intracellular domains of Unc5 and Robo to the extracellular domain of Drl and, along with Drl itself, assayed for their ability to repel axons (Fig. 7A-D). The Drl receptor is normally expressed by axons crossing the midline in the AC, whereas its ligand, Wnt5, is expressed in the PC. Drl prevents AC axons from entering the PC by repelling them from the Wnt5 source in the PC (Bonkowsky et al., 1999; Yoshikawa et al., 2003). When misexpressed by eagle-GAL4, Drl forces the axons of the PC-projecting EW neurons out of the PC and into the AC (Fig. $7 B)$. When either Drl-Unc5 or Drl-Robo is expressed by the EW neurons, the EWs switch their projections to the AC, indistinguishable from their behavior when expressing Drl itself (Fig. $7 C, D, J$ ). Therefore, each of the intracellular domains is capable of mediating repulsion when fused to the Drl extracellular domain. 

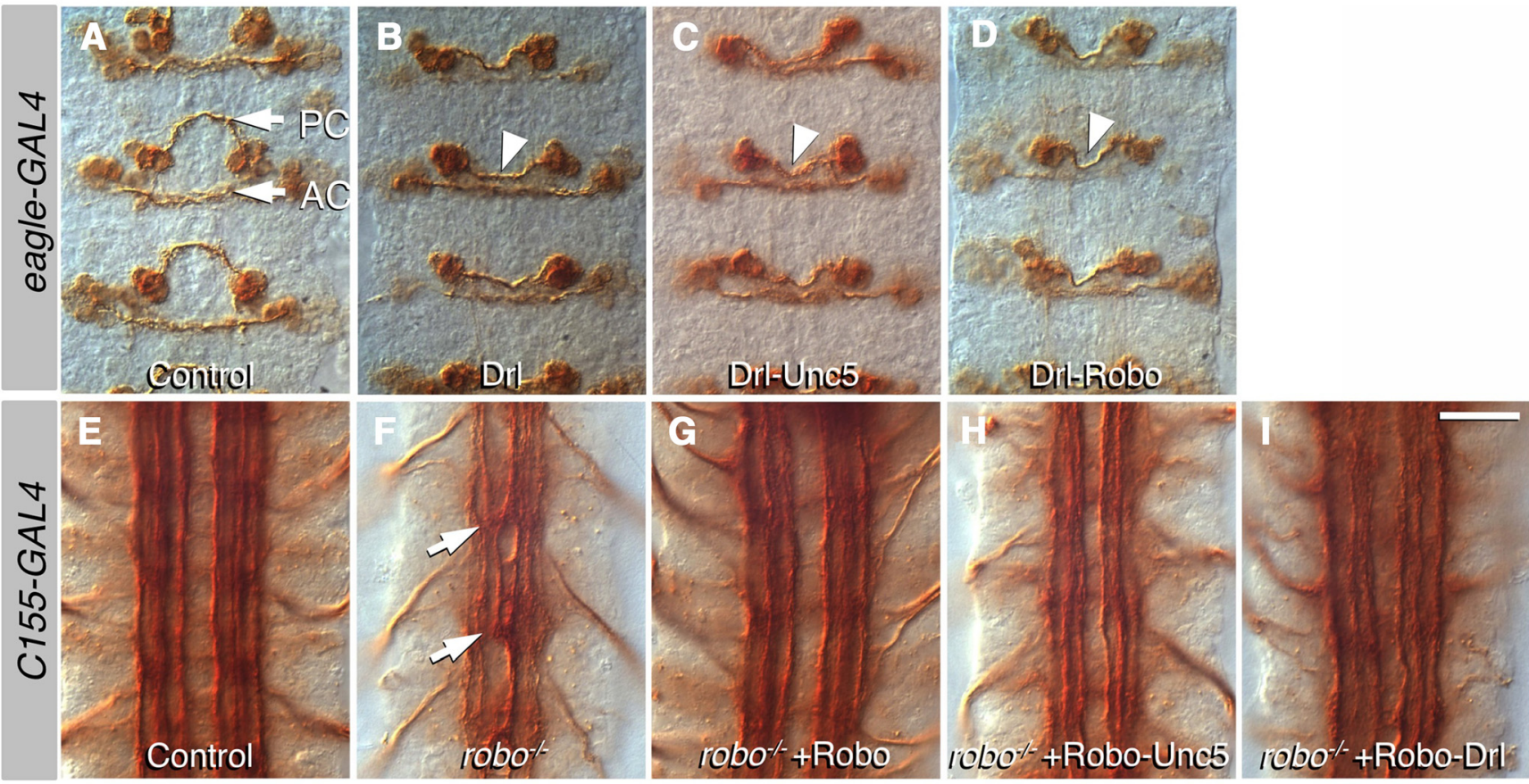

$\mathbf{J}$
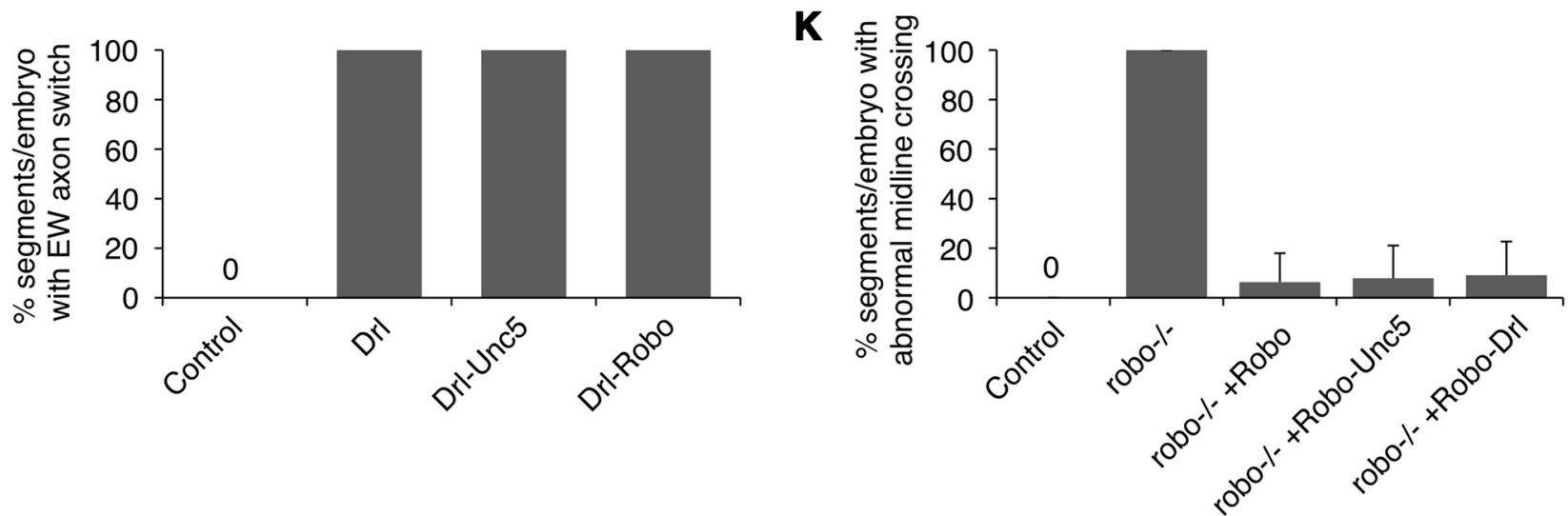

Figure 7. Repulsive receptor intracellular domains can elicit repulsion when fused to different extracellular domains. $\boldsymbol{A}-\boldsymbol{D}$, Unc5 and Robo intracellular domains mediate repulsion when fused to the Drl extracellular domain. In the control $(\boldsymbol{A})$, EW axons cross the midline in the PC, whereas the EG axons cross in the AC. When Drl (B), Drl-Unc5 (C), or Drl-Robo (D) are expressed in eagle neurons, the EW axons are repelled from the $\mathrm{PC}$ and cross the midline in the $\mathrm{AC}$ (arrowheads). Quantification shown in $J . E-I$, Unc 5 and Drl intracellular domains fused to Robo extracellular domains can rescue robo mutant midline crossing phenotype. Embryonic VNCS stained with anti-Fasll antibody. In controls (E), Fasll-positive longitudinal axons do not cross the midline, whereas in robo mutants $(\boldsymbol{F})$, longitudinal axons frequently cross the midline (arrows). Pan-neuronal expression of Robo $(\boldsymbol{G})$, Robo-Unc5 $(\boldsymbol{H})$, or Robo-Drl $(\boldsymbol{I})$ is able to rescue longitudinal axon crossing defects of robo mutants. Quantification shown in $\boldsymbol{K}$. Number of embryos examined, percentage of embryos showing phenotype, number of segments examined: $J$, Control $(n=10,0 \%, n=82)$, Drl $(n=16$, $100 \%, n=111)$, Drl-Unc5 $(n=48,100 \%, n=217)$, Drl-Robo $(n=46,100 \%, n=150)$. In $K$, Control $(n=23,0 \%, n=164)$, robo $^{-1-}(n=17,100 \%, n=147)$, robo ${ }^{-1-}+$ Robo $(n=15$, $33 \%, n=88)$, robo $^{-1-}+$ Robo-Unc5 $(n=16,38 \%, n=121)$, robo ${ }^{-1-}+$ Robo-Drl $(n=12,42 \%, n=96)$. Genotypes: A, UAS-tau-myc-GFP/+; eagle-GAL4/+;B, UAS-tau-myc-GFP/UAS-drl;

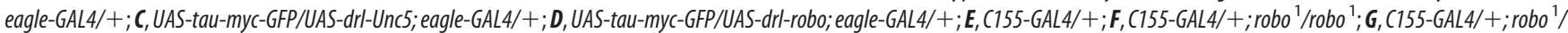
$\mathrm{robo}^{1}$; UAS-robo/+; H, C155-GAL4/+; robo ${ }^{1} / \mathrm{robo}^{1}$; UAS-robo-UnC5/+;I,C155-GAL4/+; robo $1 /$ robo $^{1}$; UAS-robo-drl/+. Scale bar, $20 \mu \mathrm{m}$.

We also fused the Unc5 and Drl intracellular domains to the Robo extracellular domain and, along with Robo itself, assayed for rescue of robo mutant defects. In robo mutants, many longitudinally projecting axons abnormally cross the midline (Fig. $7 F$ ). As shown previously (Kidd et al., 1998), pan-neuronal expression of Robo largely rescues these midline-crossing defects (Fig. 7G). Both Robo-Unc5 and Robo-Drl are capable of rescuing the robo mutant midline defects in a manner similar to Robo (Fig. $7 \mathrm{H}, I, K)$.

\section{Trio modulates Unc5, Unc5-Robo, and Unc5-Drl} repulsive signaling

Ultimately, axon guidance events, including repulsion, involve cytoskeletal rearrangements that lead to changes in growth cone orientation. To begin identifying the signaling pathway(s) coupling Unc5, Robo, and Drl to the cytoskeleton, we examined the effects of reducing putative signaling components on the activity of Unc5 and the chimeras. The guanine nucleotide exchange factor Trio has been shown to mediate axon guidance events by regulating the activity of Rac GTPases, which in turn regulate the actin cytoskeleton (Awasaki et al., 2000; Bateman et al., 2000; Newsome et al., 2000; Hakeda-Suzuki et al., 2002). To examine the role of Trio in axon repulsion, we expressed low and medium levels of Unc5, Unc5-Robo, or Unc5-Drl in the dMP2 neuron in trio mutants and assayed for any modification of the dMP2 phenotypes. We first reduced zygotic trio by $50 \%$ and observed no effect on repulsion elicited by Unc5, Unc5-Robo, or Unc5-Drl (data not shown). Because there is a maternal contribution of 


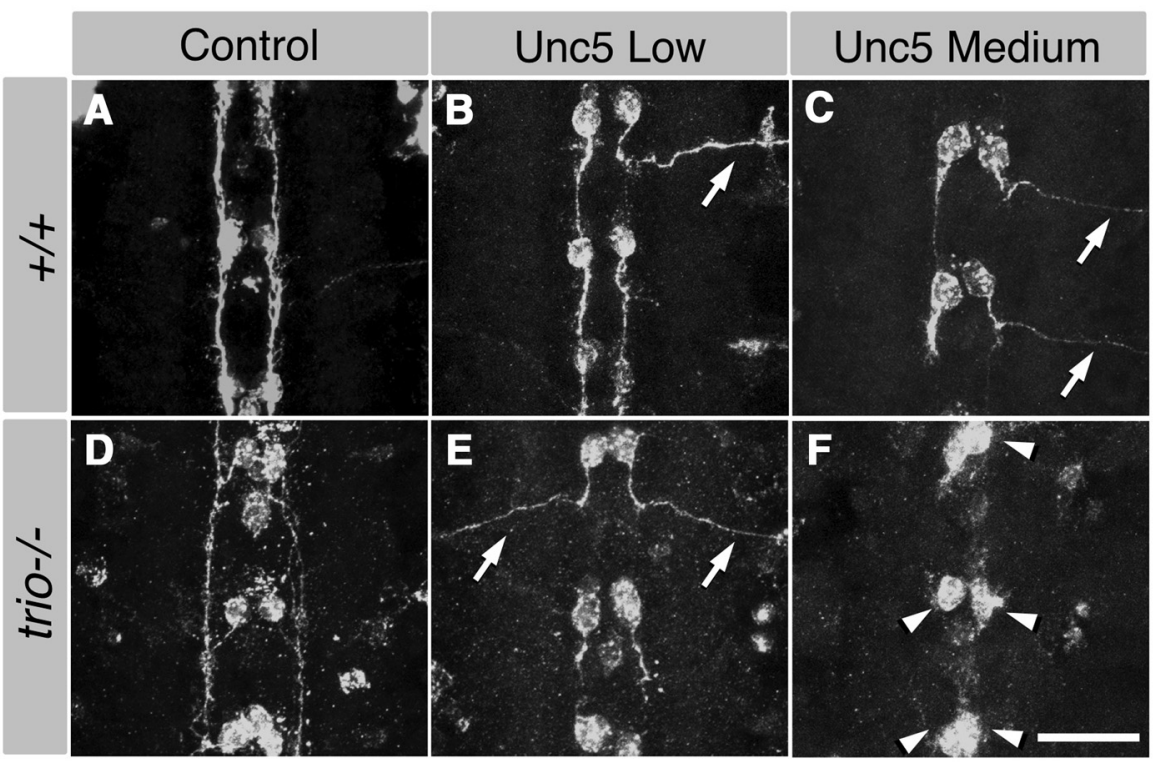

G

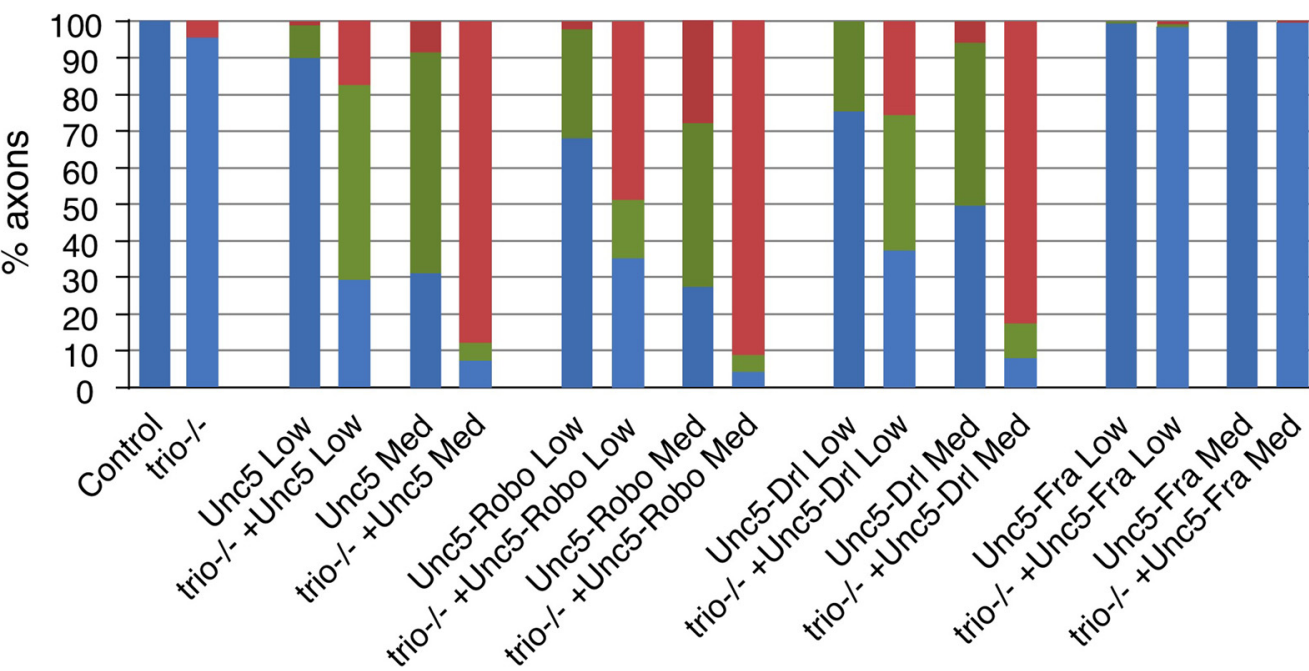

Stalled/no axon extended

Exit VNC

Project posteriorly

Figure 8. Removal of trio dramatically enhances the repulsive responses generated by Unc5, Unc5-Robo, and Unc5-Drl. $\boldsymbol{A}-\boldsymbol{F}$, dMP2 phenotypes at late stage. In wild-type background ( $\boldsymbol{A}-\boldsymbol{C}$ ), compared with the control $(\boldsymbol{A})$, low-level Unc5 expression $(\boldsymbol{B})$ drives small number of axons out of the VNC (arrow), whereas medium-level Unc5 expression ( $\boldsymbol{C}$ ) forces more axons out of the VNC (arrows). In trio mutants (D), dMP2 axons project similarly as in the control $(\boldsymbol{A})$. In embryos with low-level Unc5 expression, trio mutant can raise the number of exiting axons (arrows) to resemble that of medium Unc5 expressor in a wild-type background (cf. $\boldsymbol{E}, \boldsymbol{C}$. In embryos with medium-level Unc5 expression, trio mutant causes severe stalling phenotype ( $\boldsymbol{F}$, arrowheads). $\boldsymbol{G}$, Quantification of dMP2 axon phenotype for low and medium Unc5, Unc5-Robo, Unc5-Drl, and Unc5-Fra expressions with or without trio mutant background. Number of embryos examined, percentage of embryos showing phenotype, number of hemisegments examined: Control $(n=36,0 \%, n=200)$, trio $^{-1-}(n=11,9 \%, n=106)$, Unc5 Low $(n=10,60 \%, n=96)$, trio ${ }^{-1-}+$ Unc5 Low $(n=9,100 \%$, $n=96), \operatorname{Unc5} \operatorname{Med}(n=49,100 \%, n=416)$, trio $^{-l-}+\operatorname{Unc5} \operatorname{Med}(n=12,100 \%, n=201), \operatorname{Unc5}-\operatorname{Robo} \operatorname{Low}(n=14,100 \%, n=138)$, trio $^{-l-}+$ Unc5-Robo Low $(n=19,100 \%, n=304)$, Unc5-Robo Med $(n=54,100 \%, n=512)$, trio ${ }^{-1-}+$ Unc5-Robo Med $(n=24,100 \%, n=384)$, Unc5-Drl Low $(n=47,100 \%, n=624)$, trio ${ }^{-1-}+$ Unc5-Drl Low ( $\left.n=20,100 \%, n=270\right)$, Unc5-Drl Med $(n=102,100 \%, n=1072)$, trio ${ }^{-1-}+\operatorname{Unc5-DrlMed}(n=21,100 \%, n=381)$, Unc5-Fra Low $(n=40,3 \%, n=160)$, trio ${ }^{-1-}+$ Unc5-Fra Low $(n=7,14 \%, n=131)$, Unc5-Fra Med $(n=16,0 \%, n=144)$, trio ${ }^{-1-}+$ Unc5-Fra Med $(n=19,5 \%, n=162)$. Genotypes: receptor transgenes in the wild-type background are indicated in Figure 3 legend. Control: $d M P 2-G A L 4$ UAS-GAP-myc-GFP/+. trio ${ }^{-1-}$ : dMP2-GAL4 UAS-GAP-myc-GFP/+; trio ${ }^{\text {S137203 } / \text { trio }}{ }^{6 \mathrm{~A}}$. Receptor transgenes in trio ${ }^{-/-}$background: trio ${ }^{5137203} /$ trio $^{6 \mathrm{~A}}$ plus one copy of dMP2-GAL4, one copy of UAS-GAP-myc-GFP and one copy of the corresponding receptor transgene with low or medium expression levels. Scale bar, $20 \mu \mathrm{m}$.

trio, we were likely only modestly reducing Trio levels in these experiments. We therefore examined the effects of removing all zygotic trio by generating individuals expressing receptors in a trio mutant background. Removing zygotic trio in an otherwise wild-type background had little effect on dMP2 axons (Fig. $8 D, G)$. In contrast, removal of zygotic trio potently enhanced low-level Unc5, Unc5-Robo, and Unc5-Drl phenotypes to resemble those of medium-level expressors (Unc5 is shown in Fig. $8 E$; quantification for Unc5, Unc5-Robo, and Unc5-Drl is shown in Fig. 8G). Similarly, removal of zygotic trio enhanced medium level phenotypes to the point of causing severe axon stalling phe- notypes, similar to but more extreme than those elicited by high levels of repulsive receptor expression (Fig. $8 F, G$ ). Importantly, this enhancement is specific for the repulsive responses because removal of zygotic trio had no effect on dMP2 neurons expressing Unc5-Fra (Fig. 8G). These results argue that the repulsive actions of Unc5, Robo, and Drl involve the activity of Trio.

\section{Discussion}

To examine the responses elicited by different intracellular domains of axon guidance receptors mediating repulsion and to establish a system in which we could begin identifying down- 
stream cytoplasmic signaling molecules, we created chimeras using the extracellular and intracellular domains of Unc5, Robo, and Drl and expressed them in defined sets of neurons. By expressing Unc5-Robo, Unc5-Drl, and Unc5 itself in dMP2 neurons, the repulsion elicited by each intracellular domain could be compared directly in a single cell responding to the Netrin source in an otherwise wild-type nervous system. Given that the three intracellular domains are unrelated by sequence, we fully expected to find distinct differences in the responses elicited by each. Surprisingly, however, we found that their responses, when adjusted for levels of expression, were indistinguishable from one another. Furthermore, the responses elicited by the intracellular domains when fused to either the Drl or Robo extracellular domains were also indistinguishable from the native receptors. These results suggest that the intracellular domains of all three receptors converge onto a single pathway that mediates repulsion. We envision that different adaptors link each intracellular domain to the common signaling pathway. Although it is clear from carefully designed gene swaps between Robo receptors that, even within a single receptor family, one member cannot perfectly substitute for another (Spitzweck et al., 2010), in terms of the ability to repel axons from a ligand source, the intracellular domains of the three receptors tested here behave in an identical manner, suggesting that any differences between the receptors lie beyond the mechanics of generating repulsion.

When expressed at high levels, Unc5, Unc5-Robo, and Unc5Drl cause dMP2 axon stalling, resulting in a lack of axons being elongated at all in many cases. Importantly, this lack of axon elongation is entirely dependent upon Netrin because the phenotype is completely suppressed in a Netrin mutant background. Therefore, the stalling is not due simply to high receptor concentration nonspecifically interfering with axon growth or guidance, but rather to high levels of Netrin-dependent repulsive signaling. Conversely, high levels of Unc5-Fra do not result in any axon stalling, even when Netrins are present, indicating that the stalling is specific to the repulsive intracellular domains.

The axon-stalling phenotype suggests that high receptor density obliterates the ability of the growth cone to respond to the Netrin gradient. We envision that, with low to medium levels of receptor density, the growth cone is able to detect and respond to the relative proximodistal differences in Netrin concentration. For example, in response to the higher Netrin concentration on the proximal versus the distal side of the growth cone, filopodia might be removed and the growth cone edge retracted. However, at very high levels of receptor, the proximodistal difference in response within the growth cone may be obliterated, resulting in filopodial retraction over the entire surface and culminating in a "growth cone collapse" behavior exhibited by Unc5-expressing neurons in response to bath-applied Netrin (Bartoe et al., 2006). Such inability of the growth cone to respond to the Netrin gradient at high receptor expression levels could be the result of one or more of a number of alterations, including changes in receptor distribution on the growth cone surface or the transduction of an overwhelming signal within the growth cone that obliterates detection of the gradient.

We found that removal of zygotic trio, while having little effect alone on dMP2, strongly enhances the repulsive effects of Unc5 and the Unc5-Robo and Unc5-Drl chimeras, raising low-level phenotypes toward medium and medium-level phenotypes toward high, ultimately resulting in severe stalling of dMP2 neurons. Together with the finding of similar trio enhancement of Plexin B-mediated axon stalling (Hu et al., 2001), our result suggests that repulsion by Unc5, Robo, and Drl, and indeed perhaps repulsion in general, involves the activity of Trio. Because there is maternal contribution of trio, we were likely only reducing Trio levels in these experiments instead of removing it entirely. Consistent with this idea, elimination of both maternal and zygotic trio in an otherwise wild-type background results in axon stalling similar to the enhanced phenotypes of Unc5 and the repulsive chimeras (Hakeda-Suzuki et al., 2002).

There are several possibilities for the involvement of Trio in repulsion. Its activity might be modulated by the repulsive receptors themselves. Alternatively, Trio function could be involved indirectly in growth cone repulsion by positively regulating attraction, similar to its role in midline crossing (Forsthoefel et al., 2005). In this scenario, Trio reduction could result in lack of attraction, resulting in an unchecked repulsive signal. In either event, all three repulsive receptors tested here react similarly to the reduction in Trio and, although we cannot rule out the possibility that the repulsive receptors that we have tested were signaling through three separate pathways with qualitatively similar outputs, it seems likely that these receptors were engaging a common output pathway for repulsion, perhaps using receptorspecific adaptors. If this is true, then the notion of multiple repulsive pathways is simplified and the problem of how growth cones are repelled becomes more tractable.

\section{References}

Awasaki T, Saito M, Sone M, Suzuki E, Sakai R, Ito K, Hama C (2000) The Drosophila trio plays an essential role in patterning of axons by regulating their directional extension. Neuron 26:119-131. CrossRef Medline

Bartoe JL, McKenna WL, Quan TK, Stafford BK, Moore JA, Xia J, Takamiya K, Huganir RL, Hinck L (2006) Protein interacting with C-kinase 1/protein kinase Calpha-mediated endocytosis converts netrin-1-mediated repulsion to attraction. J Neurosci 26:3192-3205. CrossRef Medline

Bashaw GJ, Goodman CS (1999) Chimeric axon guidance receptors: the cytoplasmic domains of slit and netrin receptors specify attraction versus repulsion. Cell 97:917-926. CrossRef Medline

Bashaw GJ, Klein R (2010) Signaling from axon guidance receptors. Cold Spring Harb Perspect Biol 2:a001941. Medline

Bateman J, Shu H, Van Vactor D (2000) The guanine nucleotide exchange factor trio mediates axonal development in the Drosophila embryo. Neuron 26:93-106. CrossRef Medline

Bonkowsky JL, Yoshikawa S, O’Keefe DD, Scully AL, Thomas JB (1999) Axon routing across the midline controlled by the Drosophila Derailed receptor. Nature 402:540-544. CrossRef Medline

Boyle M, Nighorn A, Thomas JB (2006) Drosophila Eph receptor guides specific axon branches of mushroom body neurons. Development 133: 1845-1854. CrossRef Medline

Brand AH, Perrimon N (1993) Targeted gene expression as a means of altering cell fates and generating dominant phenotypes. Development 118: 401-415. Medline

Brankatschk M, Dickson BJ (2006) Netrins guide Drosophila commissural axons at short range. Nat Neurosci 9:188-194. CrossRef Medline

Callahan CA, Muralidhar MG, Lundgren SE, Scully AL, Thomas JB (1995) Control of neuronal pathway selection by a Drosophila receptor proteintyrosine kinase family member. Nature 376:171-174. CrossRef Medline

Callahan CA, Yoshikawa S, Thomas JB (1998) Tracing axons. Curr Opin Biol 8:582-586. Medline

Calleja M, Moreno E, Pelaz S, Morata G (1996) Visualization of gene expression in living adult Drosophila. Science 274:252-255. CrossRef Medline

Dittrich R, Bossing T, Gould AP, Technau GM, Urban J (1997) The differentiation of the serotonergic neurons in the Drosophila ventral nerve cord depends on the combined function of the zinc finger proteins Eagle and Huckebein. Development 124:2515-2525. Medline

Evans TA, Bashaw GJ (2010) Functional diversity of Robo receptor immunoglobulin domains promotes distinct axon guidance decisions. Curr Biol 20:567-572. Medline

Forsthoefel DJ, Liebl EC, Kolodziej PA, Seeger MA (2005) The Abelson tyrosine kinase, the Trio GEF and Enabled interact with the Netrin receptor Frazzled in Drosophila. Development 132:1983-1994. CrossRef Medline Groth AC, Fish M, Nusse R, Calos MP (2004) Construction of transgenic 
Drosophila by using the site-specific integrase from phage phiC31. Genetics 166:1775-1782. CrossRef Medline

Hakeda-Suzuki S, Ng J, Tzu J, Dietzl G, Sun Y, Harms M, Nardine T, Luo L, Dickson BJ (2002) Rac function and regulation during Drosophila development. Nature 416:438-442. CrossRef Medline

Hedgecock EM, Culotti JG, Hall DH (1990) The unc-5, unc-6, and unc-40 genes guide circumferential migrations of pioneer axons and mesodermal cells on the epidermis in C. elegans. Neuron 4:61-85. CrossRef Medline

Hong K, Hinck L, Nishiyama M, Poo MM, Tessier-Lavigne M, Stein E (1999) A ligand-gated association between cytoplasmic domains of UNC5 and DCC family receptors converts netrin-induced growth cone attraction to repulsion. Cell 97:927-941. CrossRef Medline

Hu H, Marton TF, Goodman CS (2001) Plexin B mediates axon guidance in Drosophila by simultaneously inhibiting active Rac and enhancing RhoA signaling. Neuron 32:39-51. CrossRef Medline

Keleman K, Dickson BJ (2001) Short- and long-range repulsion by the Drosophila Unc5 netrin receptor. Neuron 32:605-617. CrossRef Medline

Keleman K, Rajagopalan S, Cleppien D, Teis D, Paiha K, Huber LA, Technau GM, Dickson BJ (2002) Comm sorts robo to control axon guidance at the Drosophila midline. Cell 110:415-427. CrossRef Medline

Keleman K, Ribeiro C, Dickson BJ (2005) Comm function in commissural axon guidance: cell-autonomous sorting of Robo in vivo. Nat Neurosci 8:156-163. CrossRef Medline

Kidd T, Brose K, Mitchell KJ, Fetter RD, Tessier-Lavigne M, Goodman CS, Tear G (1998) Roundabout controls axon crossing of the CNS midline and defines a novel subfamily of evolutionarily conserved guidance receptors. Cell 92:205-215. CrossRef Medline

Kolodkin AL, Tessier-Lavigne M (2012) Mechanisms and molecules of neuronal wiring: a primer. Cold Spring Harb Perspect Biol 3.

Kolodziej PA, Timpe LC, Mitchell KJ, Fried SR, Goodman CS, Jan LY, Jan YN (1996) frazzled encodes a Drosophila member of the DCC immunoglobulin subfamily and is required for CNS and motor axon guidance. Cell 87:197-204. CrossRef Medline

Labrador JP, O'Keefe D, Yoshikawa S, McKinnon RD, Thomas JB, Bashaw GJ (2005) The homeobox transcription factor even-skipped regulates netrin-receptor expression to control dorsal motor-axon projections in Drosophila. Curr Biol 15:1413-1419. CrossRef Medline
Lundgren SE, Callahan CA, Thor S, Thomas JB (1995) Control of neuronal pathway selection by the Drosophila LIM homeodomain gene apterous. Development 121:1769-1773. Medline

Miguel-Aliaga I, Thor S (2004) Segment-specific prevention of pioneer neuron apoptosis by cell-autonomous, postmitotic Hox gene activity. Development 131:6093-6105. CrossRef Medline

Newsome TP, Schmidt S, Dietzl G, Keleman K, Asling B, Debant A, Dickson BJ (2000) Trio combines with dock to regulate Pak activity during photoreceptor axon pathfinding in Drosophila. Cell 101:283-294. CrossRef Medline

O’Donnell M, Chance RK, Bashaw GJ (2009) Axon growth and guidance: receptor regulation and signal transduction. Annu Rev Neurosci 32: 383-412. CrossRef Medline

O'Keefe DD, Thor S, Thomas JB (1998) Function and specificity of LIM domains in Drosophila nervous system and wing development. Development 125:3915-3923. Medline

Rubin GM, Spradling AC (1982) Genetic transformation of Drosophila with transposable element vectors. Science 218:348-353. CrossRef Medline

Seeger M, Tear G, Ferres-Marco D, Goodman CS (1993) Mutations affecting growth cone guidance in Drosophila: genes necessary for guidance toward or away from the midline. Neuron 10:409-426. CrossRef Medline

Spitzweck B, Brankatschk M, Dickson BJ (2010) Distinct protein domains and expression patterns confer divergent axon guidance functions for Drosophila Robo receptors. Cell 140:409-420. CrossRef Medline

Stein E, Zou Y, Poo M, Tessier-Lavigne M (2001) Binding of DCC by netrin-1 to mediate axon guidance independent of adenosine A2B receptor activation. Science 291:1976-1982. CrossRef Medline

Thomas JB, Bastiani MJ, Bate M, Goodman CS (1984) From grasshopper to Drosophila: a common plan for neuronal development. Nature 310: 203-207. CrossRef Medline

Yang L, Garbe DS, Bashaw GJ (2009) A frazzled/DCC-dependent transcriptional switch regulates midline axon guidance. Science 324:944-947. CrossRef Medline

Yoshikawa S, McKinnon RD, Kokel M, Thomas JB (2003) Wnt-mediated axon guidance via the Drosophila Derailed receptor. Nature 422:583-588. CrossRef Medline 